\title{
Characterizations of Regular Ordered Semirings by Ordered Quasi-Ideals
}

\author{
Pakorn Palakawong na Ayutthaya ${ }^{1,2}$ and Bundit Pibaljommee \\ ${ }^{1}$ Department of Mathematics, Faculty of Science, Khon Kaen University, Khon Kaen 40002, Thailand \\ ${ }^{2}$ Centre of Excellence in Mathematics CHE, Si Ayutthaya Road, Bangkok 10400, Thailand \\ Correspondence should be addressed to Bundit Pibaljommee; banpib@kku.ac.th
}

Received 27 October 2015; Revised 22 December 2015; Accepted 27 December 2015

Academic Editor: Howard E. Bell

Copyright (C) 2016 P. Palakawong na Ayutthaya and B. Pibaljommee. This is an open access article distributed under the Creative Commons Attribution License, which permits unrestricted use, distribution, and reproduction in any medium, provided the original work is properly cited.

\begin{abstract}
We introduce the notion of an ordered quasi-ideal of an ordered semiring and show that ordered quasi-ideals and ordered bi-ideals coincide in regular ordered semirings. Then we give characterizations of regular ordered semirings, regular ordered duo-semirings, and left (right) regular ordered semirings by their ordered quasi-ideals.
\end{abstract}

\section{Introduction}

The concept of a quasi-ideal was defined first by Steinfeld for semigroups and for rings [1-3] as a generalization of a right ideal and a left ideal. Then Iséki [4] introduced the notion of a quasi-ideal in a semiring without zero and investigated some of its properties. In 1994, Dönges [5] studied quasi-ideals of a semiring with zero, investigated connections between left (right) ideals, bi-ideals, and quasi-ideals and characterized regular semirings using their quasi-ideals. Later, Shabir et al. [6] have studied some properties of quasi-ideals, using quasiideals to characterize regular and intraregular semirings and regular duo-semirings. As a generalization of quasi-ideals of semirings the quasi-ideals of $\Gamma$-semirings were investigated by many authors; see, for example, [7-9].

In 2011, the notion of an ordered semiring was introduced by Gan and Jiang [10] as a semiring with a partially ordered relation on the semiring such that the relation is compatible to the operations of the semiring. In the paper, the concept of a left (right) ordered ideal, a minimal ordered ideal, and a maximal ordered ideal was defined. Then Mandal [11] studied fuzzy ideals in an ordered semiring with the least element zero and gave a characterization of regular ordered semirings by their fuzzy ideals.

In this paper, we introduce the notion of an ordered quasi-ideal of an ordered semiring and show that ordered quasi-ideals and ordered bi-ideals coincide in regular ordered semirings. Then characterizations of regular ordered semirings, regular ordered duo-semirings, and left (right) regular ordered semirings by their ordered quasi-ideals have been investigated.

\section{Preliminaries}

An ordered semiring is a system $(S,+, \cdot, \leq)$ consisting of a nonempty set $S$ such that $(S,+, \cdot)$ is a semiring, $(S, \leq)$ is a partially ordered set, and for any $a, b, x \in S$ the following conditions are satisfied:

(i) if $a \leq b$ then $a+x \leq b+x$ and $x+a \leq x+b$;

(ii) if $a \leq b$ then $a x \leq b x$ and $x a \leq x b$.

An ordered semiring $S$ is said to be additively commutative if $a+b=b+a$ for all $a, b \in S$. An element $0 \in S$ is said to be an absorbing zero if $0 a=0=a 0$ and $a+0=a=0+a$ for all $a \in S$. In this paper we assume that $S$ is an additively commutative ordered semiring with an absorbing zero 0 .

For any subsets $A, B$ of $S$ and $a \in S$, we denote

$$
\begin{aligned}
& (A]=\{x \in S \mid x \leq a \text { for some } a \in A\}, \\
& A B=\{a b \in S \mid a \in A, b \in B\},
\end{aligned}
$$




$$
\begin{aligned}
\sum A & =\left\{\sum_{i \in I} a_{i} \in S \mid a_{i}\right. \\
\in & A \text { and } I \text { is a finite subset of } \mathbb{N}\}, \\
\sum A B & =\left\{\sum_{i \in I} a_{i} b_{i} \in S \mid a_{i} \in A, b_{i}\right. \\
\in & B \text { and } I \text { is a finite subset of } \mathbb{N}\}, \\
\mathbb{N} a & =\sum\{a\} .
\end{aligned}
$$

Now, we mention some properties of finite sums on an ordered semiring.

Remark 1 . For any subsets $A, B$ of $S$, the following statements hold:

(i) $\Sigma(A] \subseteq(\Sigma A]$;

(ii) $\Sigma(\Sigma A)=\Sigma A$;

(iii) $A(\Sigma B) \subseteq \sum A B$ and $\left(\sum A\right) B \subseteq \sum A B$;

(iv) $\Sigma(A \Sigma B) \subseteq \Sigma A B$ and $\Sigma(\Sigma A) B \subseteq \Sigma A B$;

(v) $\Sigma(A+B)=\Sigma A+\Sigma B$.

We note that, for any $A \subseteq S, \Sigma A=A$ if and only if $A+A \subseteq$ $A((A,+)$ is a subsemigroup of $(S,+))$.

Now, we give the basic properties of the operator (] which are not difficult to verify.

Lemma 2. Let $A, B, C$ be subsets of an ordered semiring $S$. Then the following statements hold:

(i) $A \subseteq(A]$ and $((A]]=(A]$;

(ii) If $A \subseteq B$ then $(A] \subseteq(B]$;

(iii) $A(B] \subseteq(A](B] \subseteq(A B]$ and $(A] B \subseteq(A](B] \subseteq(A B]$;

(iv) $A+(B] \subseteq(A]+(B] \subseteq(A+B]$ and $(A]+B \subseteq(A]+(B] \subseteq$ $(A+B]$

(v) $A(B+C] \subseteq(A B+A C]$ and $(A+B] C \subseteq(A C+B C]$;

(vi) $(A \cup B]=(A] \cup(B]$;

(vii) $(A \cap B] \subseteq(A] \cap(B]$.

In (vii) of the above lemma, we have $(A \cap B]=(A] \cap(B]$ when $(A]=A$ and $(B]=B$.

Lemma 3. Let $S$ be an ordered semiring and $\emptyset \neq A \subseteq S$. If $A \subseteq\left(\sum A^{2}+\sum A S A\right]$ then $\Sigma A^{2} \subseteq\left(\sum A S A\right]$.
Proof. Assume that $A \subseteq\left(\Sigma A^{2}+\Sigma A S A\right]$. Then

$$
\begin{aligned}
\sum A^{2} & \subseteq \Sigma\left(\sum A^{2}+\sum A S A\right] A \\
& \subseteq \Sigma\left(\left(\sum A^{2}\right) A+\left(\sum A S A\right) A\right] \subseteq \Sigma\left(\sum A^{3}+\sum A S A\right] \\
& =\left(\Sigma\left(\sum A^{3}\right)+\Sigma\left(\sum A S A\right)\right]=\left(\sum A^{3}+\Sigma A S A\right] \\
& =(\Sigma A A A+\Sigma A S A] \subseteq(\Sigma A S A+\Sigma A S A] \\
& =(\Sigma A S A] .
\end{aligned}
$$

Definition 4 (see [10]). Let $S$ be an ordered semiring and $\emptyset \neq$ $A \subseteq S$. Then $A$ is said to be a left ordered ideal (right ordered ideal) if the following conditions are satisfied.

(1) $A$ is a left ideal (right ideal) of $S$.

(2) If $x \leq a$ for some $a \in A$ then $x \in A$ (i.e., $A=(A])$.

We call $A$ an ordered ideal if it is both left ordered ideal and right ordered ideal of $S$.

Example 5 (see [10]). Let $[0,1]$ be the unit interval of real numbers. Define binary operations $\oplus$ and $\odot$ on $[0,1]$ by letting $a, b \in[0,1]$,

$$
\begin{aligned}
& a \oplus b=\max \{a, b\}, \\
& a \odot b=\max \{a+b-1,0\},
\end{aligned}
$$

and an ordered relation $\leq$ is the natural order on real numbers. It is easy to show that $L=([0,1], \oplus, \odot, \leq)$ is an ordered semiring. Let $I=[0,1 / 2]$. Then we can prove that $I$ is an ordered ideal of $L$.

Lemma 6. Let $A$ be a nonempty subset of an ordered semiring S. Then

(i) $(\Sigma S A]$ is a left ordered ideal of $S$;

(ii) $(\Sigma A S]$ is a right ordered ideal of $S$;

(iii) ( $\Sigma S A S]$ is an ordered ideal of $S$.

Proof. (i) Let $x, y \in(\Sigma S A]$. Then $x \leq x^{\prime}$ and $y \leq y^{\prime}$ for some $x^{\prime}, y^{\prime} \in \Sigma S A$. It is clear that $x+y \leq x^{\prime}+y^{\prime} \in \Sigma S A$, and so $x+y \in(\Sigma S A]$. By Remark 1 and Lemma 2, we obtain $S(\Sigma S A] \subseteq(S \Sigma S A] \subseteq(\Sigma S S A] \subseteq(\Sigma S A]$. We have $((\Sigma S A]]=$ ( $\Sigma S A]$. Hence, $(\Sigma S A]$ is a left ordered ideal of $S$.

(ii) and (iii) can be proved similar to (i).

Corollary 7. Let $S$ be an ordered semiring. Then, for any $a \in S$,

(i) (Sa] is a left ordered ideal of $S$;

(ii) (aS] is a right ordered ideal of $S$;

(iii) $(\Sigma S a S]$ is an ordered ideal of $S$.

Let $A$ be a nonempty subset of an ordered semiring $S$. We denote $L(A), R(A)$ and $I(A)$ as the smallest left ordered ideal, right ordered ideal, and ordered ideal of $S$ containing 
$A$, respectively. In particular, we can show that if $A$ is a left ideal (right ideal, ideal) of $S$ then $(A]$ is the smallest left ordered ideal (resp., right ordered ideal and ordered ideal) of $S$ containing $A$.

Lemma 8. Let $A$ be a nonempty subset of an ordered semiring S. Then

(i) $L(A)=(\Sigma A+\Sigma S A]$;

(ii) $R(A)=(\Sigma A+\Sigma A S]$;

(iii) $I(A)=(\Sigma A+\Sigma S A+\Sigma A S+\Sigma S A S]$.

Proof. (i) Since $S$ has an absorbing zero, we have, for every $a \in A, a=a+0 \in \Sigma A+\Sigma S A \subseteq(\Sigma A+\Sigma S A]$. Hence, $A \subseteq$ $(\Sigma A+\Sigma S A]$. Let $x, y \in(\Sigma A+\Sigma S A]$. Then $x \leq x^{\prime}$ and $y \leq y^{\prime}$ for some $x^{\prime}, y^{\prime} \in \Sigma A+\Sigma S A$. Thus $x^{\prime}=a_{1}+b_{1}$ and $y^{\prime}=a_{2}+b_{2}$ for some $a_{1}, a_{2} \in \sum A$ and $b_{1}, b_{2} \in \Sigma S A$. It is easy to show that $a_{1}+a_{2} \in \Sigma A$ and $b_{1}+b_{2} \in \Sigma S A$. It follows that $x+y \leq$ $x^{\prime}+y^{\prime} \in \Sigma A+\Sigma S A$, and so $x+y \in(\Sigma A+\Sigma S A]$. By Remark 1 and Lemma 2, we obtain

$$
\begin{aligned}
S\left(\sum A+\Sigma S A\right] & \subseteq\left(S\left(\sum A+\Sigma S A\right)\right] \subseteq(S \Sigma A+S \Sigma S A] \\
& \subseteq(\Sigma S A+\Sigma S S A] \subseteq(\Sigma S A+\Sigma S A] \\
& =(\Sigma S A] \subseteq\left(\sum A+\Sigma S A\right] .
\end{aligned}
$$

Since $\left(\left(\sum A+\Sigma S A\right]\right]=\left(\sum A+\Sigma S A\right], L$ is a left ordered ideal of $S$. Let $K$ be any left ordered ideal of $S$ containing $A$. It turns out $\Sigma A \subseteq K$ and $\Sigma S A \subseteq K$, so $\Sigma A+\Sigma S A \subseteq K$. It follows that $(\Sigma A+\Sigma S A] \subseteq(K]=K$. Therefore, $\left(\sum A+\Sigma S A\right]$ is the smallest left ordered ideal of $S$ containing $A$.

(ii) and (iii) can be proved similar to (i).

As a special case of Lemma 8 , if $A=\{a\}$ then we have the following corollary.

Corollary 9. Let $S$ be an ordered semiring. Then, for any $a \in S$,

(i) $L(a)=(\mathbb{N a}+\mathrm{Sa}$;

(ii) $R(a)=(\mathbb{N a}+a S]$;

(iii) $I(a)=(\mathbb{N a}+S a+a S+\Sigma S a S]$.

An element $e$ of an ordered semiring $S$ is said to be an identity if $e a=a=a e$ for all $a \in S$. If $S$ has an identity, then we denote 1 as the identity of $S$.

It is not difficult to show that if $S$ has an identity, then $L(A)=(\Sigma S A], R(A)=\left(\sum A S\right]$ and $I(A)=(\Sigma S A S]$ for any $A \subseteq S$. In particular case, we have $L(a)=(S a], R(a)=(a S]$ and $I(a)=(\Sigma S a S]$ for any $a \in S$.

\section{Ordered Quasi-Ideals in Ordered Semirings}

Here, we present a notion of an ordered quasi-ideal of an ordered semiring. Then, in ordered semiring with an identity, we show that every ordered quasi-ideal can be expressed as an intersection of an ordered left ideal and an ordered right ideal.

Definition 10. Let $(S,+, \cdot, \leq)$ be an ordered semiring and let $(Q,+)$ be a subsemigroup of $(S,+)$. Then $Q$ is said to be an ordered quasi-ideal of $S$ if the following conditions are satisfied:

(1) $(\Sigma S Q] \cap(\Sigma Q S] \subseteq Q$;

(2) if $x \leq q$ for some $q \in Q$ then $x \in Q$ (i.e., $Q=(Q])$.

It is clear that every left ordered ideal (right ordered ideal and ordered ideal) of an ordered semiring $S$ is an ordered quasi-ideal of $S$. Moreover, each ordered quasi-ideal of $S$ is a subsemiring of $S$; indeed, $Q Q \subseteq(Q Q] \subseteq(S Q] \cap(Q S] \subseteq$ $(\Sigma S Q] \cap(\Sigma Q S] \subseteq Q$.

Example 11. Let $S=\{a, b, c, d\}$. Define binary operations + and $\cdot$ on $S$ by the following equations:

$$
\begin{array}{l|llll}
+ & a & b & c & d \\
\hline a & a & b & c & d \\
b & b & b & b & b \\
c & c & b & c & d \\
d & d & b & d & d \\
\cdot & a & b & c & d \\
\hline a & a & a & a & a \\
b & a & b & b & b \\
c & a & c & c & c \\
d & a & b & b & b
\end{array} .
$$

Then $(S,+, \cdot)$ is an additively commutative semiring with an absorbing zero $a$. Define a binary relation $\leq$ on $S$ by

$$
\leq:=\{(a, a),(b, b),(c, c),(d, d),(b, d)\} .
$$

We give the covering relation " $\prec$ " and the figure of $S$ :

$$
\prec:=\{(b, d)\}
$$

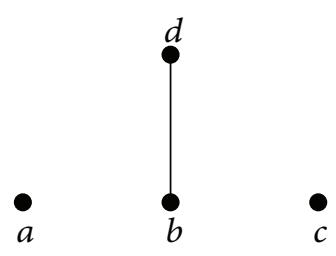

Now, $(S,+, \cdot, \leq)$ is an ordered semiring. Let $Q=\{a, b\}$. We have $(\Sigma S Q] \cap(\Sigma Q S]=\{a, b, c\} \cap\{a, b\}=Q$ and $(Q]=Q$. Hence, $Q$ is an ordered quasi-ideal of $S$ but is not a left ordered ideal of $S$, since $S Q=\{a, b, c\} \nsubseteq Q$.

Lemma 12. Let $S$ be an ordered semiring and let $\left\{Q_{i} \mid i \in I\right\}$ be a family of ordered quasi-ideals of $S$. Then $\bigcap_{i \in I} Q_{i}$ is an ordered quasi-ideal of $S$.

Let $A$ be a nonempty subset of an ordered semiring $S$. We denote $Q(A)$ the smallest ordered quasi-ideal of $S$ containing A.

Theorem 13. Let $S$ be an ordered semiring and let $A$ be a nonempty subset of $S$. Then $Q(A)=(\Sigma A+((\Sigma S A] \cap(\Sigma A S])]$. 
Proof. Let $Q=(\Sigma A+((\Sigma S A] \cap(\Sigma A S])]$. Since $S$ has an absorbing zero, we have $a=a+0 \in \Sigma A+((\Sigma S A] \cap(\Sigma A S]) \subseteq Q$ for every $a \in A$. Hence, $A \subseteq Q$. Let $x, y \in Q$. Then $x \leq x^{\prime}$ and $y \leq y^{\prime}$ for some $x^{\prime}, y^{\prime} \in \Sigma A+((\Sigma S A] \cap(\Sigma A S])$. Thus $x^{\prime}=a_{1}+b_{1}$ and $y^{\prime}=a_{2}+b_{2}$ for some $a_{1}, a_{2} \in \sum A$ and $b_{1}, b_{2} \in(\Sigma S A] \cap\left(\sum A S\right]$. Clearly, $a_{1}+a_{2} \in \sum A$ and $b_{1}+b_{2} \in(\Sigma S A] \cap(\Sigma A S]$. It follows that $x+y \leq x^{\prime}+y^{\prime} \epsilon$ $\sum A+\left(\left(\sum S A\right] \cap\left(\sum A S\right]\right)$, and so $x+y \in Q$. By Remark 1 and Lemma 2, we obtain

$$
\begin{aligned}
(\Sigma S Q] \cap(\Sigma Q S] & \subseteq(\Sigma S Q] \\
& =(\Sigma S(\Sigma A+((\Sigma S A] \cap(\Sigma A S])]] \\
& \subseteq(\Sigma S(\Sigma A+(\Sigma S A]]] \\
& \subseteq(\Sigma(S \Sigma A+S(\Sigma S A]]) \\
& \subseteq(\Sigma(\Sigma S A+(\Sigma S S A]]) \\
& \subseteq(\Sigma(\Sigma S A+\Sigma S S A]] \\
& \subseteq(\Sigma(\Sigma S A+\Sigma S A]] \subseteq(\Sigma(\Sigma S A]] \\
& \subseteq((\Sigma S A]]=(\Sigma S A] .
\end{aligned}
$$

Similarly, we can show that $(\Sigma S Q] \cap(\Sigma Q S] \subseteq(\Sigma A S]$. Thus $(\Sigma S Q] \cap(\Sigma Q S] \subseteq(\Sigma S A] \cap(\Sigma A S] \subseteq \Sigma A+((\Sigma S A] \cap(\Sigma A S]) \subseteq Q$ Since $(Q]=Q$, we obtain that $Q$ is an ordered quasi-ideal of $S$ containing $A$. Let $K$ be any ordered quasi-ideal of $S$ containing $A$. It follows that $(\Sigma S A] \cap(\Sigma A S] \subseteq(\Sigma S K] \cap(\Sigma K S] \subseteq$ $K$. So $\Sigma A+((\Sigma S A] \cap(\Sigma A S]) \subseteq K$. Hence, $Q=(\Sigma A+((\Sigma S A] \cap$ $\left.\left.\left(\sum A S\right]\right)\right] \subseteq(K]=K$. Therefore, $Q$ is the smallest ordered quasi-ideal of $S$ containing $A$.

As a special case of Theorem 13, if $A=\{a\}$ then we have the following corollary.

Corollary 14. Let $S$ be an ordered semiring. Then $Q(a)=$ $(\mathbb{N a}+((S a] \cap(a S])]$ for any $a \in S$.

If $S$ has an identity, then it is easy to check that $Q(A)=$ $(\Sigma S A] \cap(\Sigma A S]$ for any $A \subseteq S$. In particular case, we have $Q(a)=(S a] \cap(a S]$ for any $a \in S$.

Let $Q(S)$ be the set of all ordered quasi-ideals of an ordered semiring $S$. Using Lemma 12 , we define the operations $\wedge$ and $\vee$ on $Q(S)$ by letting $P_{1}, P_{2} \in \mathbb{Q}(S)$,

$$
\begin{aligned}
& P_{1} \wedge P_{2}=P_{1} \cap P_{2}, \\
& P_{1} \vee P_{2}=Q\left(P_{1} \cup P_{2}\right) .
\end{aligned}
$$

Then we obtain the following theorem.

Theorem 15. Let $S$ be an ordered semiring. Then $(Q(S), \wedge, \vee)$ is a complete lattice.

Theorem 16. The intersection of a left ordered ideal $L$ and a right ordered ideal $R$ of an ordered semiring $S$ is an ordered quasi-ideal of $S$.
Proof. It is easy to show that $L \cap R$ is a subsemigroup of $(S,+)$. By Remark 1 and Lemma 2, we obtain

$$
\begin{aligned}
& (\Sigma S(L \cap R)] \cap(\Sigma(L \cap R) S] \subseteq(\Sigma S(L \cap R)] \\
& \quad=(\Sigma(S L \cap S R)] \subseteq(\Sigma S L] \subseteq L, \\
& (\Sigma S(L \cap R)] \cap(\Sigma(L \cap R) S] \subseteq(\Sigma(L \cap R) S] \\
& \quad=(\Sigma(L S \cap R S)] \subseteq(\Sigma R S] \subseteq R .
\end{aligned}
$$

Hence, $(\Sigma S(L \cap R)] \cap(\Sigma(L \cap R) S] \subseteq L \cap R$. Let $s \in S$ such that $s \leq x$ for some $x \in L \cap R$. Then $s \in(L \cap R] \subseteq(L] \cap(R]=$ $L \cap R$.

The converse of Theorem 16 is not true as Example 2.1 page 8 in [2] given by A. H. Clifford.

Corollary 17. Let $S$ be an ordered semiring. Then the following statements hold.

(i) $(\Sigma S A] \cap(\Sigma A S]$ is an ordered quasi-ideal of $S$, for any $A \subseteq S$.

(ii) $(S a] \cap(a S]$ is an ordered quasi-ideal of $S$, for any $a \in S$.

Proof. (i) By Lemma 6, we have ( $\Sigma S A]$ and $\left(\sum A S\right]$ a left and a right ordered ideal of $S$, respectively. Then by Theorem 16, we have that $(\Sigma S A] \cap(\Sigma A S]$ is an ordered quasi-ideal of $S$.

(ii) It is a particular case of (i).

Now, we will show that the converse of Theorem 16 is true if $S$ contains an identity as the following theorem.

Theorem 18. Let $S$ be an ordered semiring with identity. Then every ordered quasi-ideal $Q$ of $S$ can be written in the form $Q=$ $R \cap L$ for some right ordered ideal $R$ and left ordered ideal $L$ of $S$.

Proof. Assume that $S$ has an identity. Let $Q$ be an ordered quasi-ideal of $S$. Then $R(Q)=(\Sigma Q S]$ and $L(Q)=(\Sigma S Q]$. We obtain $Q \subseteq R(Q) \cap L(Q)$ and $R(Q) \cap L(Q)=(\Sigma Q S] \cap(\Sigma S Q] \subseteq$ $Q$. Hence, $Q=R(Q) \cap L(Q)$.

\section{Regular Ordered Semirings}

In this section, we show that in regular ordered semirings the converse of Theorem 16 is true and ordered quasi-ideals coincide with ordered bi-ideals. Then we give characterizations of regular ordered semirings, regular ordered duo-semirings, and left regular and right regular ordered semirings by their ordered quasi-ideals.

Definition 19 (see [11]). An element $a$ of an ordered semiring $S$ is said to be regular if $a \leq a x a$ for some $x \in S$. An ordered semiring $S$ is said to be regular if every element $a \in S$ is regular.

The following lemma is characterizations of regular ordered semiring which directly follows Definition 19. 
Lemma 20. Let $S$ be an ordered semiring. Then the following statements are equivalent:

(i) $S$ is regular;

(ii) $A \subseteq(\Sigma A S A]$ for each $A \subseteq S$;

(iii) $a \in(a S a]$ for any $a \in S$.

Now, we will show that the converse of Theorem 16 is true in regular ordered semirings.

Theorem 21. Every ordered quasi-ideal of a regular ordered semiring $S$ can be written in the form $Q=R \cap L$ for some right ordered ideal $R$ and left ordered ideal $L$ of $S$.

Proof. Let $Q$ be an ordered quasi-ideal of $S$. By Lemma 8, we have $R(Q)=(\Sigma Q+\Sigma Q S]$ and $L(Q)=(\Sigma Q+\Sigma S Q]$. Now, $Q \subseteq$ $R(Q) \cap L(Q)$. Let $q \in Q$. Since $S$ is regular, there exists $x \in S$ such that $q \leq q x q \in \Sigma Q S$. So $Q \subseteq(\Sigma Q S]$. Since $Q+Q \subseteq Q$, $\Sigma Q=Q$. It follows that

$$
\begin{aligned}
(\Sigma Q S] & \subseteq(\Sigma Q+\Sigma Q S]=(Q+\Sigma Q S] \subseteq((\Sigma Q S]+\Sigma Q S] \\
& \subseteq(\Sigma Q S] .
\end{aligned}
$$

This implies that $R(Q)=(\Sigma Q S]$. Similarly, we can show that $L(Q)=(\Sigma S Q]$. Hence, $R(Q) \cap L(Q)=(\Sigma Q S] \cap(\Sigma S Q] \subseteq Q$. Therefore, $Q=R(Q) \cap L(Q)$.

Definition 22. Let $(S,+, \cdot, \leq)$ be an ordered semiring. A subsemigroup $(B,+)$ of $(S,+)$ is said to be an ordered bi-ideal of $S$ if the following conditions hold:

(1) $B S B \subseteq B$;

(2) if $x \leq b$ for some $b \in B$, then $x \in B$ (i.e., $B=(B])$.

We note that condition (1) of Definition 22 is equivalent to $\Sigma B S B \subseteq B$.

Theorem 23. Every ordered quasi-ideal of an ordered semiring $S$ is an ordered bi-ideal of $S$.

Proof. Let $Q$ be an ordered quasi-ideal of $S$. Then $\Sigma Q S Q \subseteq$ $\Sigma Q S \subseteq(\Sigma Q S]$ and $Q S Q \subseteq \Sigma S Q \subseteq(\Sigma S Q]$. So, $\Sigma Q S Q \subseteq$ $(\Sigma S Q] \cap(\Sigma Q S] \subseteq Q$. Hence, $Q$ is an ordered bi-ideal of $S$.

The converse of Theorem 23 is not generally true as the following example.

Example 24. Let $S=\{a, b, c, d, e\}$. Define binary operations + and by the following equations:

$$
\begin{array}{c|lllll}
+ & a & b & c & d & e \\
\hline a & a & b & c & d & e \\
b & b & b & d & d & d \\
c & c & d & d & d & d \\
d & d & d & d & d & d \\
e & e & d & d & d & e
\end{array},
$$

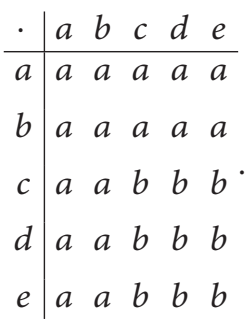

Then $(S,+, \cdot)$ is an additively commutative semiring with an absorbing zero $a$. Define a binary relation $\leq$ on $S$ by

$$
\begin{aligned}
\leq & :=\{(a, a),(b, b),(c, c),(d, d),(e, e),(a, b),(a, c),(a, e), \\
& (a, d),(b, d),(c, d),(e, d)\} .
\end{aligned}
$$

We give the covering relation " $\prec$ " and the figure of $S$ :

$$
\prec:=\{(a, b),(a, c),(a, e),(b, d),(c, d),(e, d)\} .
$$

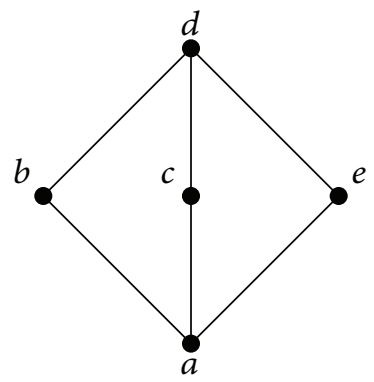

Then $(S,+, \cdot, \leq)$ is an ordered semiring but not regular, since $d \not d x d$ for any $x \in S$. Let $B=\{a, e\}$. It is easy to show that $B$ is an ordered bi-ideal but not an ordered quasi-ideal of $S$, since $(\Sigma S B] \cap(\Sigma B S]=\{a, b\} \nsubseteq B$.

Now, we show that in regular ordered semirings, ordered bi-ideals and ordered quasi-ideals coincide as the following theorem.

Theorem 25. Let $S$ be a regular ordered semiring. Then ordered bi-ideals and ordered quasi-ideals coincide in $S$.

Proof. By Theorem 23, we have that every ordered quasiideal of $S$ is an ordered bi-ideal of $S$. Now, we show that every ordered bi-ideal of $S$ is an ordered quasi-ideal of $S$. Let $B$ be an ordered bi-ideal of $S$. Let $a \in(\Sigma S B] \cap(\Sigma B S]$. By Lemma 20, Remark 1, and Lemma 2, we obtain a $\epsilon$ $(a S a] \subseteq((\Sigma B S] S(\Sigma S B]] \subseteq((\Sigma B S S](\Sigma S B]] \subseteq((\Sigma B S)(\Sigma S B)] \subseteq$ $(\Sigma(B S(\Sigma S B))] \subseteq(\Sigma(\Sigma B S S B)] \subseteq(\Sigma B S B] \subseteq B$. Hence, $B$ is an ordered quasi-ideal of $S$.

Theorem 26. Let $S$ be an ordered semiring. Then the following statements are equivalent:

(i) $S$ is regular;

(ii) $(\Sigma R L]=R \cap L$ for every right ordered ideal $R$ and left ordered ideal $L$ of $S$;

(iii) $B=(\Sigma B S B]$ for each ordered bi-ideal $B$ of $S$;

(iv) $Q=(\Sigma Q S Q]$ for each ordered quasi-ideal $Q$ of $S$. 
Proof. (i) $\Rightarrow$ (ii): assume that $S$ is regular and let $R$ and $L$ be a right ordered ideal and a left ordered ideal of $S$, respectively. So, $\left(\sum R L\right] \subseteq(\Sigma R]=R$ and $(\Sigma R L] \subseteq(\Sigma L]=L$. Hence, $\left(\sum R L\right] \subseteq R \cap L$. Let $a \in R \cap L$. Since $S$ is regular, $a \leq a x a$ for some $x \in S$. Since $a \in R, x a \in L$. It follows that $a \leq a(x a) \in R L$. This means $a \in(R L] \subseteq(\Sigma R L]$. Therefore, $\left(\sum R L\right]=R \cap L$.

(ii) $\Rightarrow$ (iii): assume that (ii) holds. Let $B$ be an ordered bi-ideal of $S$. It is clear that $(\Sigma B S B] \subseteq B$. By assumption, $B \subseteq R(B) \cap L(B)=(\Sigma R(B) L(B)]$. By Lemma 8, Remark 1, and Lemmas 2 and 3, we have

$$
\begin{aligned}
B & \subseteq(\Sigma B+\Sigma B S] \cap(\Sigma B+\Sigma S B] \\
& =(\Sigma((\Sigma B+\Sigma B S](\Sigma B+\Sigma S B])] \\
& \subseteq(\Sigma((\Sigma B+\Sigma B S)(\Sigma B+\Sigma S B))] \\
& \subseteq(\Sigma(\Sigma B(\Sigma B+\Sigma S B)+\Sigma B S(\Sigma B+\Sigma S B))] \\
& \subseteq\left(\Sigma\left(\Sigma B^{2}+\Sigma B S B+\Sigma B S B+\Sigma B S S B\right)\right] \\
& \subseteq\left(\Sigma\left(\Sigma B^{2}+\Sigma B S B\right)\right]=\left(\Sigma\left(\Sigma B^{2}\right)+\Sigma(\Sigma B S B)\right] \\
& =\left(\Sigma B^{2}+\Sigma B S B\right] \subseteq((\Sigma B S B]+\Sigma B S B] \subseteq(\Sigma B S B] .
\end{aligned}
$$

(iii) $\Rightarrow$ (iv): it follows from Theorem 23 .

(iv) $\Rightarrow$ (i): let $a \in S$. Then $Q(a)=(Q(a) S Q(a)]$. By Corollary 14, Remark 1, and Lemma 2, we have

$$
\begin{aligned}
a & \in(\mathbb{N} a+((S a] \cap(a S])] \\
& =\left(\sum((\mathbb{N} a+((S a] \cap(a S])] S(\mathbb{N} a+((S a] \cap(a S])])\right] \\
& \subseteq\left(\sum((\mathbb{N} a+(a S]] S(\mathbb{N} a+(S a]])\right] \\
& \subseteq\left(\sum((\mathbb{N} a+a S] S(\mathbb{N} a+S a])\right] \\
& \subseteq\left(\sum(((\mathbb{N a}+a S) S](\mathbb{N} a+S a])\right] \\
& \subseteq\left(\sum((a S](\mathbb{N a}+S a])\right] \subseteq\left(\sum(a S(\mathbb{N} a+S a))\right] \\
& \subseteq\left(\sum a S a\right]=(a S a] .
\end{aligned}
$$

By Lemma 20, $S$ is regular.

Theorem 27. Let $S$ be a regular ordered semiring. Then the following statements hold:

(i) every ordered quasi-ideal $Q$ of $S$ can be written in the form $Q=R \cap L=(R L]$ for some right ordered ideal $R$ and left ordered ideal $L$ of $S$;

(ii) $\left(Q^{2}\right]=\left(Q^{3}\right]$ for each ordered quasi-ideal $Q$ of $S$.

Proof. (i) It is obvious by Theorems 21 and 26.

(ii) Let $Q$ be an ordered quasi-ideal of $S$. Clearly, $((Q Q) Q] \subseteq(Q Q]$. Let $x \in(Q Q]$. Then $x \leq q_{1} q_{2}$ for some $q_{1}, q_{2} \in Q$. Since $S$ is regular, there exists $s \in S$ such that $x \leq$ $q_{1} q_{2} \leq\left(q_{1} q_{2}\right) s\left(q_{1} q_{2}\right) \in Q Q S Q Q$. Hence, $x \in(Q(Q S Q) Q] \subseteq$ (QQQ]. Therefore, $\left(Q^{2}\right]=\left(Q^{3}\right]$.

Theorem 28. Let $S$ be an ordered semiring. Then $S$ is regular if and only if $B \cap I \cap L \subseteq(B I L]$ for every ordered bi-ideal $B$, every ordered ideal $I$, and every left ordered ideal $L$ of $S$.
Proof. Let $B, I$, and $L$ be an ordered bi-ideal, an ordered ideal, and a left ordered ideal of $S$, respectively. Let $a \in B \cap I \cap L$. Since $S$ is regular, $a \leq a x a \leq$ axaxaxa $\in B I L$. Hence, $B \cap I \cap L \subseteq$ $(B I L]$.

Conversely, assume that $B \cap I \cap L \subseteq(B I L]$ for every ordered bi-ideal $B$, every ordered ideal $I$, and every left ordered ideal $L$ of $S$. Then we obtain $R \cap L=R \cap S \cap L \subseteq(R S L] \subseteq(R L] \subseteq\left(\sum R L\right]$ for every right ordered ideal $R$ and left ordered ideal $L$ of $S$. On the other hand, we have $(\Sigma R L] \subseteq R \cap L$. Hence, $(\Sigma R L]=R \cap L$. By Theorem 26, $S$ is regular.

Definition 29. An ordered semiring $S$ is said to be an ordered duo-semiring if every one-sided (right or left) ordered ideal of $S$ is an ordered ideal of $S$.

We note that every multiplicatively commutative ordered semiring is an ordered duo-semiring, but the converse is not generally true. Now, we give an example of a multiplicatively noncommutative ordered semiring which is an ordered duosemiring.

Example 30. Let $S=\{a, b, c, d, e\}$. Define binary operations + and by the following equations:

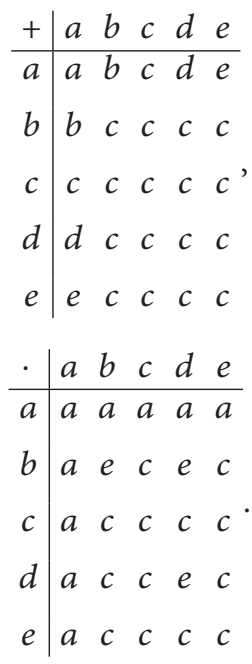

Then $(S,+, \cdot)$ is an additively commutative semiring with an absorbing zero $a$. Define a binary relation $\leq$ on $S$ by

$$
\leq:=\{(a, a),(b, b),(c, c),(d, d),(e, e),(e, c)\} .
$$

We give the covering relation " $\prec$ ” and the figure of $S$ :

$$
\prec:=\{(e, c)\} .
$$

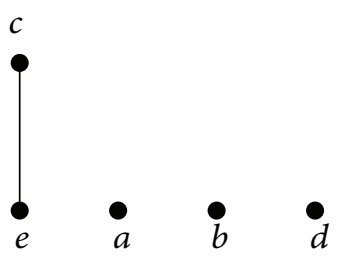

Then $(S,+, \cdot, \leq)$ is an ordered semiring which is not multiplicatively commutative, since $b d \neq d b$. We have all one-sided ordered ideals of $S$ which are as follows:

$$
\{a\},\{a, c\},\{a, c, e\},\{a, b, c, e\},\{a, c, d, e\}, S .
$$


It is not difficult to check that all of them are ordered ideals of $S$. This shows that $S$ is an ordered duo-semiring.

Lemma 31. Let $S$ be an ordered semiring. Then the following conditions are equivalent:

(i) $S$ is an ordered duo-semiring;

(ii) $R(A)=L(A)$ for each $A \subseteq S$;

(iii) $R(a)=L(a)$ for each $a \in S$.

Proof. (i) $\Rightarrow$ (ii) and (ii) $\Rightarrow$ (iii) are obvious.

(iii) $\Rightarrow$ (i): let $L$ be a left ordered ideal of $S$ and let $x \in L, s \in S$. By assumption, we have $x s \in R(x) S \subseteq R(x)=$ $L(x) \subseteq L(L)=L$. It follows that $L$ is a right ordered ideal of $S$. Similarly, we have that every right ordered ideal of $S$ is a left ordered ideal of $S$. Hence, $S$ is an ordered duo-semiring.

Theorem 32. Let $S$ be an ordered duo-semiring. Then $S$ is regular if and only if $\left(\Sigma Q_{1} Q_{2}\right]=Q_{1} \cap Q_{2}$ for each two ordered quasi-ideals $Q_{1}$ and $Q_{2}$ of $S$.

Proof. Assume that $S$ is a regular ordered semiring. Let $Q_{1}$ and $Q_{2}$ be ordered quasi-ideals of $S$. By Theorem 21, $Q_{1}$ and $Q_{2}$ can be written in the forms

$$
\begin{aligned}
& Q_{1}=R_{1} \cap L_{1}, \\
& Q_{2}=R_{2} \cap L_{2}
\end{aligned}
$$

for some $R_{1}, R_{2}$ and $L_{1}, L_{2}$ which are right ordered ideals and left ordered ideals of $S$, respectively. Since $S$ is an ordered duosemiring, $R_{1}, R_{2}, L_{1}$, and $L_{2}$ are ordered ideals of $S$. It follows that $Q_{1}$ and $Q_{2}$ are ordered ideals of $S$. By Theorem 26, we have $\left(\Sigma Q_{1} Q_{2}\right]=Q_{1} \cap Q_{2}$.

Conversely, assume that $\left(\Sigma Q_{1} Q_{2}\right]=Q_{1} \cap Q_{2}$ for each two ordered quasi-ideals $Q_{1}$ and $Q_{2}$ of $S$. Let $A \subseteq S$. By assumption, $A \subseteq Q(A) \cap Q(A)=(\Sigma Q(A) Q(A)]$. By Theorem 13, Remark 1, and Lemmas 2 and 3, we have

$$
\begin{aligned}
& A \subseteq(\Sigma((\Sigma A+((\Sigma S A] \cap(\Sigma A S])] \\
& \cdot(\Sigma A+((\Sigma S A] \cap(\Sigma A S])])] \subseteq(\Sigma((\Sigma A+(\Sigma A S]] \\
& \left.\left.\cdot\left(\sum A+(\Sigma S A]\right]\right)\right] \subseteq\left(\sum\left(\left(\sum A+\sum A S\right]\left(\sum A+\Sigma S A\right]\right)\right] \\
& \subseteq\left(\sum\left(\left(\sum A+\sum A S\right)\left(\sum A+\Sigma S A\right)\right)\right] \\
& \subseteq(\Sigma(\Sigma A(\Sigma A+\Sigma S A)+\Sigma A S(\Sigma A+\Sigma S A))] \\
& \subseteq\left(\Sigma\left(\Sigma A^{2}+\Sigma A S A+\Sigma A S A+\Sigma A S S A\right)\right] \\
& \subseteq\left(\Sigma\left(\sum A^{2}+\Sigma A S A\right)\right]=\left(\Sigma\left(\Sigma A^{2}\right)+\Sigma(\Sigma A S A)\right] \\
& =\left(\Sigma A^{2}+\Sigma A S A\right] \subseteq((\Sigma A S A]+\Sigma A S A] \subseteq(\Sigma A S A] \text {. }
\end{aligned}
$$

By Lemma 20, $S$ is a regular ordered semiring.

Theorem 33. Let $S$ be an ordered duo-semiring. Then the following conditions are equivalent:

(i) $S$ is regular; (ii) $\left(\Sigma L_{1} L_{2}\right]=L_{1} \cap L_{2}$ and $\left(\Sigma R_{1} R_{2}\right]=R_{1} \cap R_{2}$ for each two left ordered ideals $L_{1}, L_{2}$ and right ordered ideals $R_{1}, R_{2}$ of $S$;

(iii) $(\Sigma R L]=R \cap L=(\Sigma L R]$, for each right ordered ideal $R$ and left ordered ideal $L$ of $S$.

Proof. It is obvious by Theorem 26.

Definition 34. Let $S$ be an ordered semiring. Then an element $a \in S$ is said to be left regular (right regular) if $a \leq x a^{2}$ ( $a \leq$ $a^{2} x$ ) for some $x \in S$. An ordered semiring $S$ is said to be left regular (right regular) if every element $a \in S$ is left regular (right regular).

Example 35. Let $S=\{a, b, c, d, e, f\}$. Define binary operations + and $\cdot$ on $S$ by the following equations:

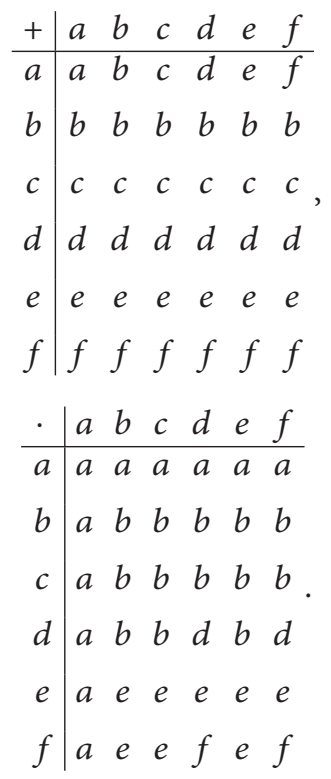

Then $(S,+, \cdot)$ is a semiring with an absorbing zero $a$. Define a binary relation $\leq$ on $S$ by

$$
\begin{aligned}
\leq & :=\{(a, a),(b, b),(c, c),(d, d),(e, e),(f, f),(b, c), \\
& (b, d),(b, e),(b, f),(c, d),(c, e),(c, f),(d, f),(e, f)\} .
\end{aligned}
$$

We give the covering relation " $\prec$ " and the figure of $S$ :

$$
\prec:=\{(b, c),(c, d),(c, e),(d, f),(e, f)\} .
$$

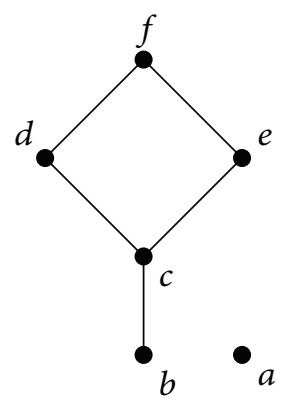

Now, $(S,+, \cdot, \leq)$ is an ordered semiring. Clearly, $a, b, d, e$, and $f$ are left regular. We consider $c \leq e c^{2}=e b=e$. This implies 
that $S$ is left regular. Since there does not exist $x \in S$ such that $c \leq c x c, S$ is not regular.

Example 36. Consider the ordered semiring $S=(\mathbb{N} \cup$ $\{0\}, \max , \min , \leq)$ where $\leq$ is the natural order relation on numbers. Since $n \leq \min \{n, n, n\}$ for any $n \in \mathbb{N}$, we get $S$ a regular, left regular, and right regular ordered semiring.

The following lemmas can be easily proved using Definition 34 .

Lemma 37. Let $S$ be an ordered semiring. Then the following statements are equivalent:

(i) S is left regular;

(ii) $A \subseteq\left(\Sigma S A^{2}\right]$ for each $A \subseteq S$;

(iii) $a \in\left(S a^{2}\right]$ for each $a \in S$.

Lemma 38. Let $S$ be an ordered semiring. Then the following statements are equivalent:

(i) $S$ is right regular;

(ii) $A \subseteq\left(\Sigma A^{2} S\right]$ for each $A \subseteq S$;

(iii) $a \in\left(a^{2} S\right]$ for each $a \in S$.

Definition 39. Let $T$ be a nonempty subset of an ordered semiring $S$. Then $T$ is said to be semiprime if for any $a \in S$, if $a^{2} \in T$, then $a \in T$.

We note that a nonempty subset $T$ of $S$ is semiprime if and only if, for any $\emptyset \neq A \subseteq S, A^{2} \subseteq T$ implies $A \subseteq T$. Because, if $T$ is semiprime, $\emptyset \neq A \subseteq T$, and $a \in A$ then $a^{2} \in T$ and so $a \in T$; that is, $A \subseteq T$.

Theorem 40. Let $S$ be an ordered semiring. Then $S$ is left regular and right regular if and only if every ordered quasi-ideal of $S$ is semiprime.

Proof. Let $Q$ be an ordered quasi-ideal of $S$. Let $A$ be a nonempty subset of $S$ such that $A^{2} \subseteq Q$. Since $S$ is left regular and by Lemma 37, we have $A \subseteq\left(\Sigma S A^{2}\right]$. Since $S$ is right regular and by Lemma 38, we have $A \subseteq\left(\Sigma A^{2} S\right]$. Hence, $A \subseteq\left(\Sigma S A^{2}\right] \cap\left(\Sigma A^{2} S\right] \subseteq(\Sigma S Q] \cap(\Sigma Q S] \subseteq Q$. Therefore, $Q$ is semiprime.

Conversely, assume that every ordered quasi-ideal of $S$ is semiprime. Let $A \subseteq S$. By Theorem 13, we have $Q\left(A^{2}\right)=$ $\left(\Sigma A^{2}+\left(\left(\Sigma S A^{2}\right] \cap\left(\Sigma A^{2} S\right]\right)\right]$. Since $A^{2} \subseteq Q\left(A^{2}\right)$ is semiprime, $A \subseteq Q\left(A^{2}\right)=\left(\sum A^{2}+\left(\left(\sum S A^{2}\right] \cap\left(\sum A^{2} S\right]\right)\right]$. Then we obtain

$$
\begin{aligned}
A & \subseteq\left(\sum A^{2}+\left(\left(\sum S A^{2}\right] \cap\left(\sum A^{2} S\right]\right)\right] \\
& \subseteq\left(\Sigma A^{2}+\left(\Sigma S A^{2}\right]\right] \subseteq\left(\Sigma A^{2}+\Sigma S A^{2}\right], \\
A & \subseteq\left(\Sigma A^{2}+\left(\left(\Sigma S A^{2}\right] \cap\left(\sum A^{2} S\right]\right)\right] \\
& \subseteq\left(\Sigma A^{2}+\left(\Sigma A^{2} S\right]\right] \subseteq\left(\Sigma A^{2}+\Sigma A^{2} S\right] .
\end{aligned}
$$

In case $A \subseteq\left(\Sigma A^{2}+\Sigma S A^{2}\right]$, we get

$$
\begin{aligned}
\Sigma A^{2} & \subseteq \Sigma A\left(\Sigma A^{2}+\Sigma S A^{2}\right] \subseteq \Sigma\left(A \Sigma A^{2}+A \Sigma S A^{2}\right] \\
& \subseteq \Sigma\left(\Sigma A^{3}+\Sigma A S A^{2}\right] \subseteq \Sigma\left(\Sigma A^{3}+\Sigma S A^{2}\right] \\
& \subseteq\left(\Sigma\left(\Sigma A^{3}\right)+\Sigma\left(\Sigma S A^{2}\right)\right] \subseteq\left(\Sigma A^{3}+\Sigma S A^{2}\right] \\
& \subseteq\left(\Sigma S A^{2}+\Sigma S A^{2}\right]=\left(\Sigma S A^{2}\right]
\end{aligned}
$$

and so $A \subseteq\left(\Sigma A^{2}+\Sigma S A^{2}\right] \subseteq\left(\left(\Sigma S A^{2}\right]+\Sigma S A^{2}\right] \subseteq\left(\Sigma S A^{2}\right]$. By Lemma 37, $S$ is left regular. Similarly, in case $A \subseteq\left(\sum A^{2}+\right.$ $\left.\Sigma A^{2} S\right]$, we get $A \subseteq\left(A^{2} S\right]$. By Lemma $38, S$ is right regular.

\section{Conflict of Interests}

The authors declare that there is no conflict of interests regarding the publication of this paper.

\section{Acknowledgment}

This work has been supported by the Centre of Excellence in Mathematics, the Commission on Higher Education, Thailand.

\section{References}

[1] O. Steinfeld, "On ideal-quotients and prime ideals," Acta Mathematica Academiae Scientiarum Hungaricae, vol. 4, pp. 289-298, 1953.

[2] O. Steinfeld, Quasi-Ideals in Rings and Semigroups, vol. 10 of Disquisitiones Mathematicae Hungaricae, Akadémiai Kiadó, Budapest, Hungary, 1978.

[3] O. Steinfeld, "Über die Quasiideale von Halbgruppen," Publicationes Mathematicae Debrecen, vol. 4, pp. 262-275, 1956.

[4] K. Iséki, "Quasi-ideals in semirings without zero," Proceedings of the Japan Academy, vol. 34, pp. 79-81, 1958.

[5] C. Dönges, "On Quasi-ideals of semirings," International Journal of Mathematics and Mathematical Sciences, vol. 17, no. 1, pp. 47-58, 1994.

[6] M. Shabir, A. Ali, and S. Batool, "A note on quasi-ideals in semirings," Southeast Asian Bulletin of Mathematics, vol. 27, no. 5, pp. 923-928, 2004.

[7] R. Chinram, "A note on quasi-ideals in $\Gamma$-semirings," International Mathematical Forum, vol. 3, no. 25-28, pp. 1253-1259, 2008.

[8] R. Chinram, "A note on quasi-ideals in regular $\Gamma$-semirings," International Journal of Contemporary Mathematical Sciences, vol. 3, no. 35, pp. 1725-1731, 2008.

[9] R. D. Jagatap and Y. S. Pawar, "Quasi-ideals and minimal quasiideals in $\Gamma$-semirings," Novi Sad Journal of Mathematics, vol. 39, no. 2, pp. 79-87, 2009.

[10] A. P. Gan and Y. L. Jiang, "On ordered ideals in ordered semirings," Journal of Mathematical Research \& Exposition, vol. 31, no. 6, pp. 989-996, 2011.

[11] D. Mandal, "Fuzzy ideals and fuzzy interior ideals in ordered semirings," Fuzzy Information and Engineering, vol. 6, no. 1, pp. 101-114, 2014. 


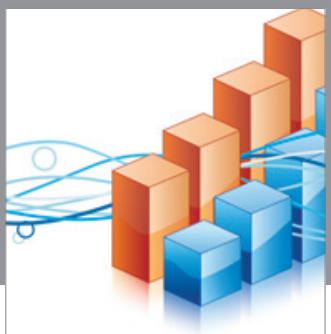

Advances in

Operations Research

vatem alat4

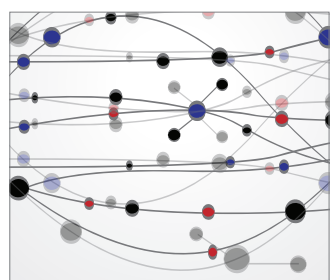

\section{The Scientific} World Journal
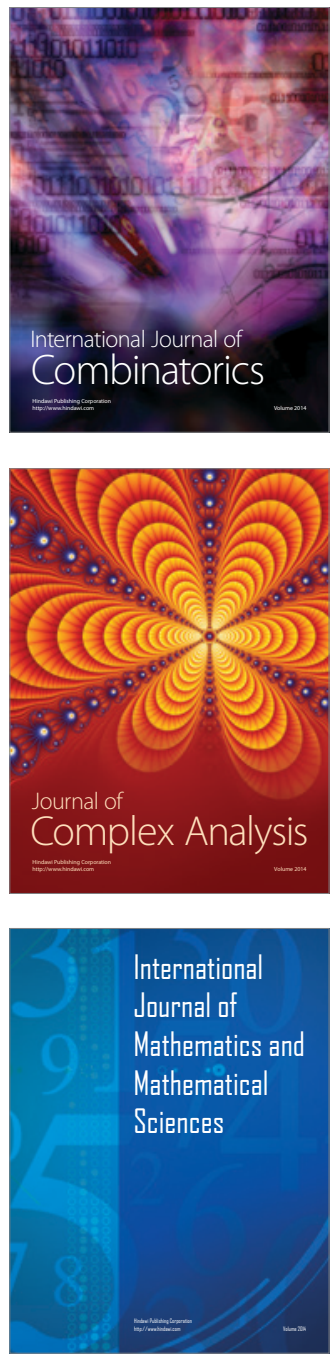
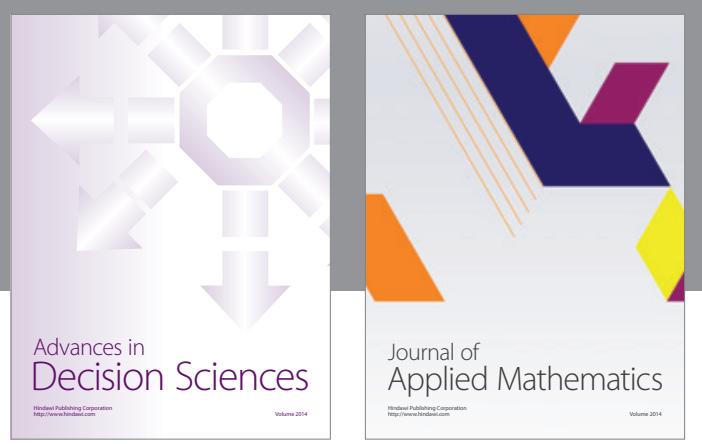

Algebra

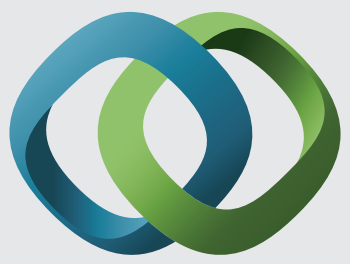

\section{Hindawi}

Submit your manuscripts at

http://www.hindawi.com
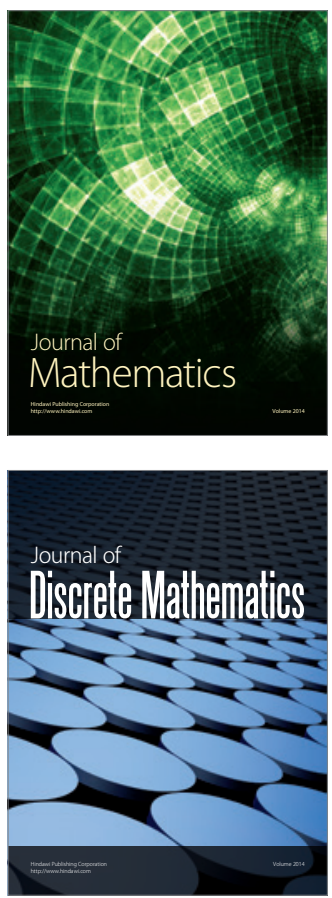

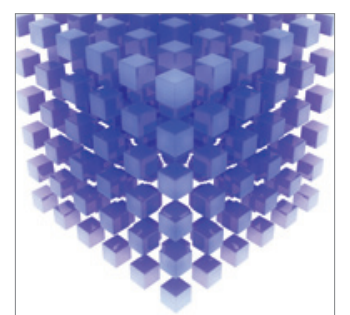

Mathematical Problems in Engineering
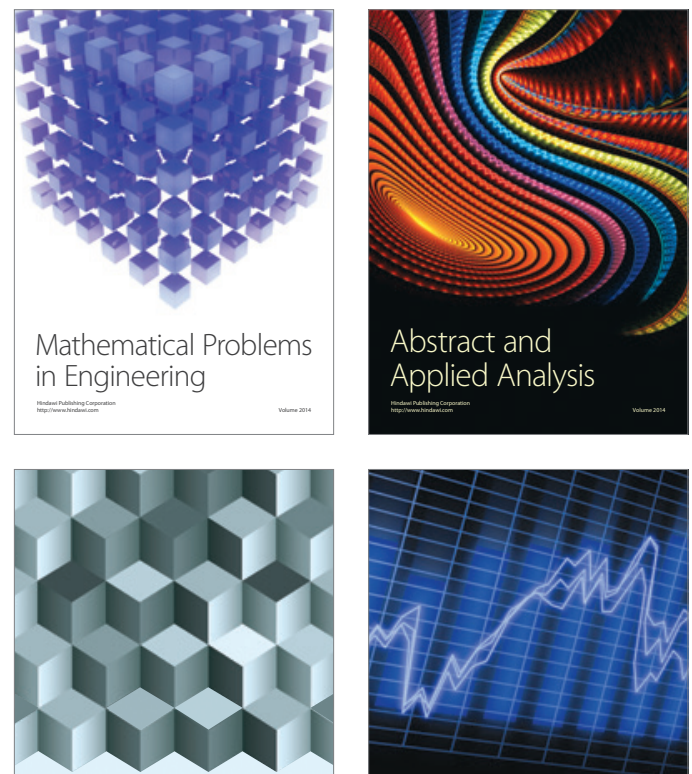

Journal of

Function Spaces

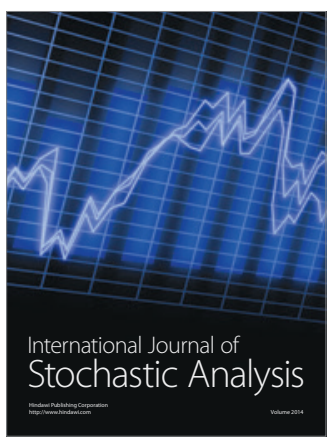

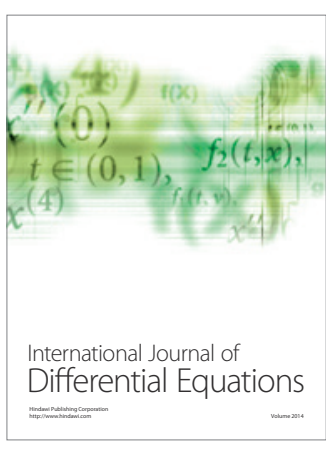
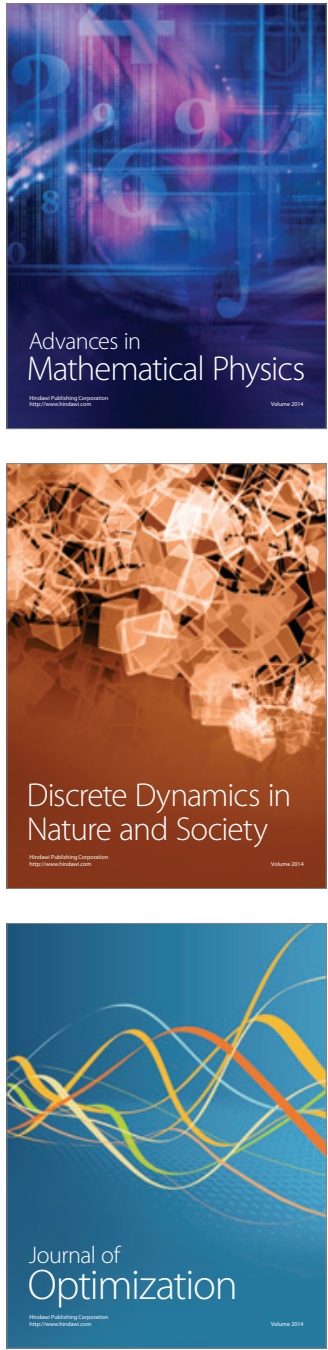